\title{
Celecoxib attenuates cachectic events in mice by modulating the expression of vascular endothelial growth factor
}

\author{
XIAOYA XU, MAN JIANG, YING ZHANG, YULI BI and MINGYONG HAN \\ Cancer Therapy and Research Center, Shandong Provincial Hospital, \\ Shandong University, Jinan, Shandong 250021, P.R. China
}

Received December 1, 2013; Accepted July 21, 2014

DOI: $10.3892 / \mathrm{mmr} .2014 .2730$

\begin{abstract}
Chronic inflammation is one of the main symptoms of cancer cachexia, and cyclooxygenase 2 inhibitors, such as celecoxib, may be beneficial in counteracting the major symptoms of this syndrome. In the current study, celecoxib was orally administered to BALB/c male mice with colon 26 adenocarcinoma. Tumor growth, survival rate, body weight and food intake of the mice with cancer cachexia were recorded during the experiments. The host inflammatory response was assessed by morphological observations and hematoxylin-eosin staining. The serum levels of vascular endothelial growth factor (VEGF), granulocyte-macrophage colony-stimulating factor, interleukin- 6 and tumor necrosis factor- $\alpha$ in mice with cancer cachexia were measured by ELISA. Celecoxib administration attenuated the decline in body weight and food intake of mice with cancer cachexia, and improved the survival rate of cachectic mice. Erythrocyte counts and hemoglobin concentration significantly increased in cachectic mice receiving celecoxib compared with control cachectic mice. Notably, celecoxib administration significantly reduced the serum level of VEGF in mice with colon 26 adenocarcinoma, and the cachectic events were also relieved by treatment with a VEGF antibody. The cyclooxygenase 2 inhibitor celecoxib produced positive therapeutic effects in mice with cancer cachexia. This function was regulated at least partly by downregulation of serum levels of VEGF.
\end{abstract}

\section{Introduction}

Cachexia is a significant cause of morbidity and mortality in patients with advanced cancer, occurring in up to $80 \%$ of cases $(1,2)$. Weight loss, anorexia, inflammation, insulin resistance and increased muscle protein breakdown are

Correspondence to: Professor Mingyong Han, Cancer Therapy and Research Center, Shandong Provincial Hospital, Shandong University, 324 Jingwuweiqi Road, Jinan, Shandong 250021, P.R. China

E-mail: hanmingyong71@126.com

Key words: cancer cachexia, celecoxib, cyclooxygenase 2 inhibitor, vascular endothelial growth factor frequently associated with cachexia (3). Although the precise mechanism of cancer cachexia has not been fully elucidated, it is now clear that the persistent inflammatory response of the host, in conjunction with inappropriate production and release of cytokines, such as tumor necrosis factor (TNF)- $\alpha$, interleukin (IL)-1 and IL-6, are central to the pathogenesis of this disease $(4,5)$. There is increasing evidence that eicosanoids, such as prostaglandins have an important function in the development of cachexia $(6,7)$. Non-steroidal anti-inflammatory drugs (NSAIDs) have been demonstrated to block the protein-catabolizing effects that occur in cachectic mice and prevent muscle protein breakdown in tumor-bearing rats $(8,9)$.

Cyclooxygenases (COXs) are key rate-limiting enzymes in the conversion of arachidonic acid to prostaglandins. There are at least two isoforms of cyclooxygenase, including COX-1 and COX-2 (10). COX-1 is constitutively expressed in numerous types of cell and is important in homeostasis, whereas COX-2 is usually absent but inducible by various cytokines, growth factors and mitogens. Overexpression of COX-2 has been demonstrated in tissues from several types of tumor tissue and in various cell lines $(11,12)$. Selective COX-2 inhibitors exhibit marked antineoplastic activity in a number of model tumor systems (13-15). It has also been observed that acute treatment of severely cachectic tumor-bearing animals with the COX-2 inhibitor celecoxib maintained or reduced the serum calcium level and produced a rapid and significant weight gain, compared with tumor-matched vehicle-treated animals (16). The studies suggested that COX-2 inhibitors may be a potential treatment approach to cachexia; however, little is known about the underlying mechanisms.

In the present study, the colon 26 carcinoma mouse model of cachexia, a well-studied model of cancer cachexia, was used with the aim to investigate the possible effects of celecoxib on cancer cachexia progression and to elucidate the complex involvement of cytokines. The results demonstrated that the reduction of serum vascular endothelial growth factor (VEGF) concentration was associated with COX-2 inhibition. These findings suggest a novel mechanism by which a COX-2 inhibitor ameliorates cancer cachexia.

\section{Materials and methods}

Cell culture. Colon 26 cells were maintained in Dulbecco's modified Eagle's medium supplemented with $10 \%$ fetal bovine 
serum, sodium pyruvate, non-essential amino acids and L-glutamine (Gibco-BRL, Paisley, UK) in a $37^{\circ} \mathrm{C}, 5 \% \mathrm{CO}_{2}$ humidified incubator. Colon 26 cells were harvested from subconfluent cultures with $0.25 \%$ trypsin (Hyclone, Logan, UT, USA) and resuspended in phosphate-buffered saline (PBS; Sigma, Santa Clara, CA, USA) at $10^{7}$ cells $/ \mathrm{ml}$ for injection, prior to use.

Animals. Seven-week-old male BALB/c mice were purchased from the Center for New Drug Evaluation (Shandong University, Jinan, China) and housed in a barrier system with controlled light (12/12-h light/dark cycle) and temperature $\left(21-22^{\circ} \mathrm{C}\right)$. All animal experiments were conducted in accordance with the guidelines for animal experimentation of the Shandong Provincial Animal Board (Jinan, China).

Mouse model of cancer cachexia. Tumor cells were subcutaneously implanted at a density of $\sim 1 \times 10^{6}$ subcutaneously in the backs of the BALB/c mice following anesthesia, which was induced and maintained by inhalation of halothane (Sigma). All experimental animals were observed to have a palpable tumor within five days of the start of the experiment. Treatment was initiated when tumors reached a mean volume of $0.1 \mathrm{ml}$, according to a previous study (; the selective COX-2 inhibitor celecoxib (250 mg/kg/day; Pfixer, New York, NY, USA) was administered in the diet. This treatment was maintained for the duration of the experiment. The VEGF-neutralizing antibody bevacizumab $(5 \mathrm{mg} / \mathrm{kg}$; Roche Diagnostics, Basel, Switzerland) was intraperitoneally injected twice per week, with a total of three injections administered to each mouse. Body weight was measured three times a week. Each animal group was administered $150 \mathrm{~g}$ food, the following day the remaining food was weighed and the reduction in weight was divided by the number of mice to determine the average food intake per animal. Tumor growth was measured every three days and tumor volume was calculated as follows: Tumor volume $=$ length $\times(\text { width })^{2} \times 0.52$.

Tissue and organ collection. Tumor-bearing and control mice were sacrificed at different time points. At the time of sacrifice, animals were anesthesized with halothane and exsanguinated via closed cardiac puncture, blood was allowed to clot for $2 \mathrm{~h}$ at room temperature then centrifuged for $20 \mathrm{~min}$ at $2,000 \mathrm{x} \mathrm{g}$. Serum was removed and stored at $-80^{\circ} \mathrm{C}$ until required. Whole blood $(100 \mu \mathrm{l})$ was prepared with EDTA (Sigma) as an anticoagulant to determine hematological parameters. Primary tumors were excised, the liver, kidneys and spleen were resected and weighed, and were then immediately fixed with $10 \%$ paraformaldehyde overnight, followed by washing with PBS. A fraction of the tissues and organs was embedded with paraffin.

Determination of circulating cytokines. Cytokine levels in serum samples were assessed using the Quantikine ELISA kits (R\&D Systems, Inc., Minneapolis, MN, USA).

Histological studies. Malignant and non-malignant paraffin-embedded tissues were cut to a thickness of $4 \mu \mathrm{m}$ for hematoxylin-eosin (H\&E) staining. Slides were examined under a light microscope (DM500; Leica, Mannheim, Germany) and images were captured at a magnification x 200 .
A

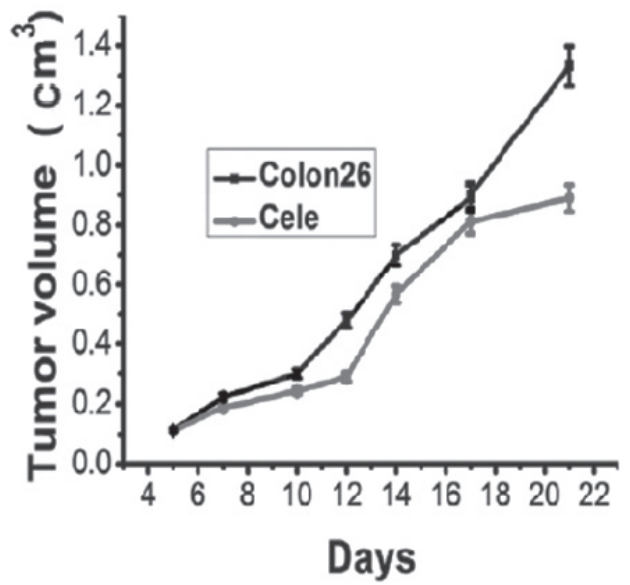

B

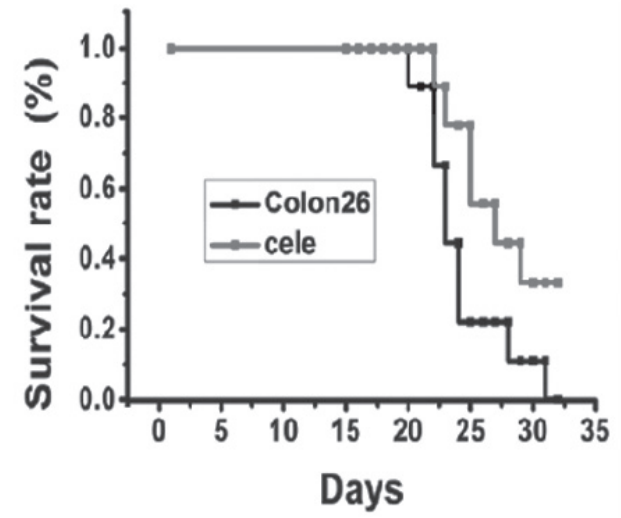

Figure 1. (A) Tumor volume and (B) survival rate of mice following injection of colon 26 cells. Celecoxib delayed tumor growth and increased the survival rate. Colon 26, control mice injected with colon 26 cancer cells; cele, mice injected with colon 26 cancer cells and treated with celecoxib.

Statistical analysis. Results are expressed as the mean \pm standard deviation. Analyses of different treatment groups were conducted using Student's t-test in the SPSS statistical software package, version 16.0 (SPSS, Inc., Chicago, IL, USA). P<0.05 was considered to indicate a statistically significant difference. A Kaplan-Meier survival curve was generated using Statistica, version 5.0 (Statsoft, Inc., Tulsa, OK, USA).

\section{Results}

Celecoxib reduces tumor growth and increases survival rate in mice with cancer cachexia. Tumor appearances were observed on day five, and the volumes of the tumors increased until the day on which the mice were sacrificed. Fig. 1A shows a representative tumor growth curve of the colon 26 tumor group compared with the celecoxib treatment group. The celecoxib-treated group presented delayed tumor growth compared with the control group. There was a significant reduction in tumor volume between the treated and non-treated groupon day 21 (Fig. 1A, P<0.05). Mortality occurred in mice injected with colon 26 tumors $\sim 21$ days following tumor injection, and the survival rate reduced to 0 on day 32 . Between days 23 and 32 post-tumor inoculation, treatment of tumor bearing mice with celecoxib led to a significant increase in survival rate compared with that of the control tumor-bearing mice (Fig. 1B). 
Celecoxib reduces weight loss and increases food intake in mice with cancer cachexia. Body weight and food intake are considered to be indicators of cancer cachexia in animal models. No significant difference was identified among groups in initial body weight and food intake. The body weight of mice carrying colon 26 tumors started to reduce around day nine post-inoculation. From day 12 to 21 post-inoculation, prior to the occurrence of cachexia-mediated mortality of tumor-bearing mice, the body weights of mice receiving celecoxib treatment were identified to be significantly greater than those of tumor-bearing control mice (Fig. 2A). The average body weight of mice at the time of tumor inoculation was $18.7 \mathrm{~g}$, this then reduced to $14.6 \mathrm{~g}$ in tumor-bearing control mice on day 21 , while it reduced to $17.8 \mathrm{~g}$ in the mice receiving daily celecoxib treatment. Food intake was similar in the two groups prior to day seven (Fig. 2B). However, a significant reduction in food intake was observed from day eight in the control group, which was significantly ameliorated by celecoxib treatment.

Celecoxib ameliorates anemia in mice with cancer cachexia. Hematological analysis of peripheral blood indicated a significant reduction in hematocrit at day 21 post colon 26 tumor implantation. The level of hemoglobin and the number of erythrocytes in peripheral blood were significantly reduced. These results demonstrate that colon 26 tumor-bearing mice suffered from severe anemia. Erythrocyte count and hemoglobin concentration in the celecoxib-treated group were significantly higher than those of the tumor-bearing control mice on day 21 post-inoculation (Table I).

Celecoxib reduces host inflammatory response in mice with cancer cachexia. The injection of colon 26 cells into mice resulted in increases in the volumes of liver, lung and spleen. These increases were reduced by oral administration of celecoxib. Histological examination demonstrated inflammatory changes in the organs of colon 26 tumor-bearing mice. Following treatment with celecoxib, $H \& E$ staining indicated a marked reduction in inflammatory cell infiltration in the lung, liver and spleen (Fig. 3).

Celecoxib alters the expression of inflammatory cytokines in mice with cancer cachexia. Serum concentrations of VEGF, granulocyte-macrophage colony-stimulating factor (GM-CSF), IL-6 and TNF- $\alpha$ were measured by ELISA in the sera of the controls and celecoxib-treated tumor mice. As presented in Fig. 4, the levels of IL-6 and VEGF were reduced significantly by oral administration of celecoxib, while levels of TNF- $\alpha$ and GM-CSF were not affected by the administration of celecoxib.

Anti-VEGF ameliorates cachexia events in mice with cancer cachexia. In order to investigate whether an anti-VEGF agent has the ability to reverse cachexia in mice injected by colon 26 tumor cells, a neutralizing anti-mouse VEGF antibody (bevacizumab) was administered to the tumor-bearing mice for 10 days. The tumor induced an anemic phenotype, including paleness of the paws, which was reverted by anti-VEGF treatment (Fig. 5A). The erythrocyte count and hemoglobin concentration in the bevacizumab group were significantly
Table I. Effect of celecoxib on hemograms of colon 26-adenocarcinoma model mice.

\begin{tabular}{lcc}
\hline Group & $\begin{array}{c}\text { Control } \\
\text { mice }\end{array}$ & $\begin{array}{c}\text { Celecoxib } \\
\text { treated mice }\end{array}$ \\
\hline Erythrocyte $\left(\times 10^{12} / 1\right)$ & $5.4 \pm 0.7$ & $7.6 \pm 0.4^{\mathrm{b}}$ \\
Hemoglobin $(\mathrm{g} / \mathrm{l})$ & $11.4 \pm 1.6$ & $13.7 \pm 2.1^{\mathrm{a}}$ \\
Platelets $\left(\mathrm{x} 10^{9} / \mathrm{l}\right)$ & $727 \pm 23.1$ & $769.5 \pm 37.2$ \\
White blood cells $\left(\mathrm{x} 10^{9} / 1\right)$ & $5.7 \pm 0.4$ & $5.9 \pm 0.7$
\end{tabular}

Data are expressed as the mean \pm standard deviation; $n=7$ in each group. ${ }^{\mathrm{a}} \mathrm{P}<0.05$ vs. the control group; ${ }^{b} \mathrm{P}<0.01 \mathrm{vs}$. the control group.
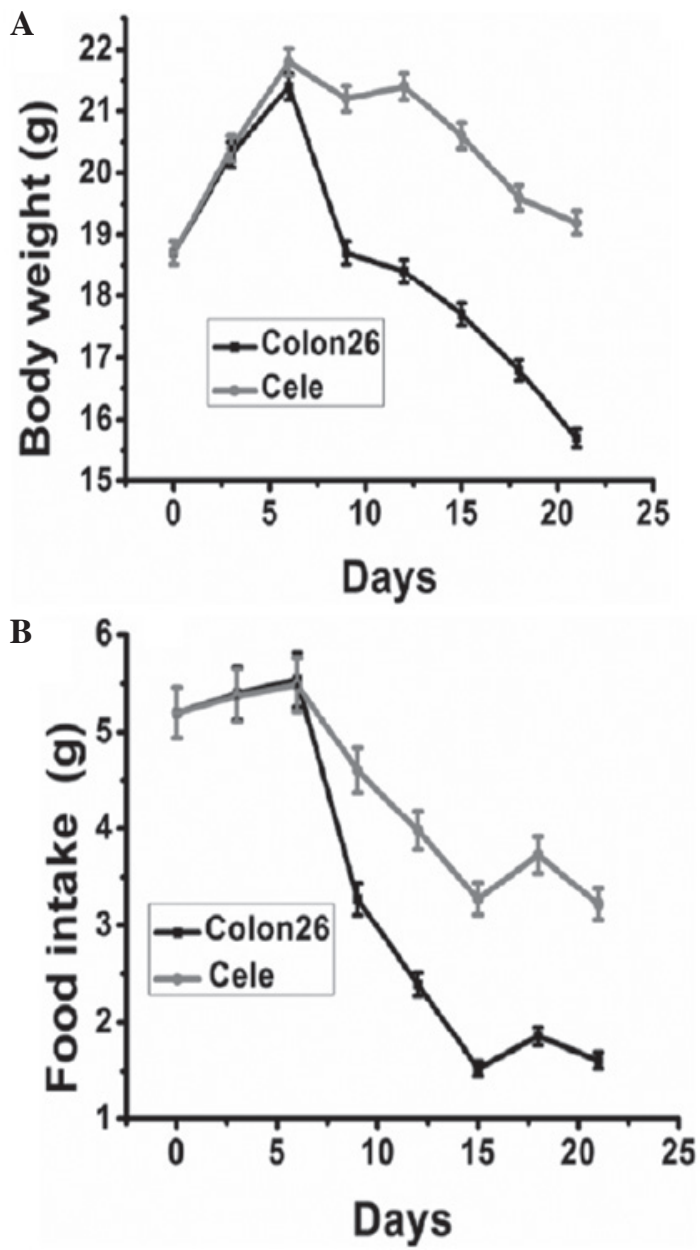

Figure 2. Time-course alterations in (A) body weight and (B) food intake of tumor-bearing control and celecoxib-treated mice, measured every three days. The body weights of mice receiving celecoxib were significantly heavier than those of the colon 26 tumor group $(\mathrm{P}<0.05)$. The food intake of mice receiving celecoxib was significantly greater than those of the colon 26 tumor group $(\mathrm{P}<0.05)$. Animal weight is inclusive of tumor weight. Cele, celecoxib.

higher than in untreated tumor-bearing mice on day 21 post-inoculation (Fig. 5B and C). The food intake and body weight of the mice receiving bevacizumab were significantly increased compared with the untreated tumor-bearing mice (Fig. 5D and E). 


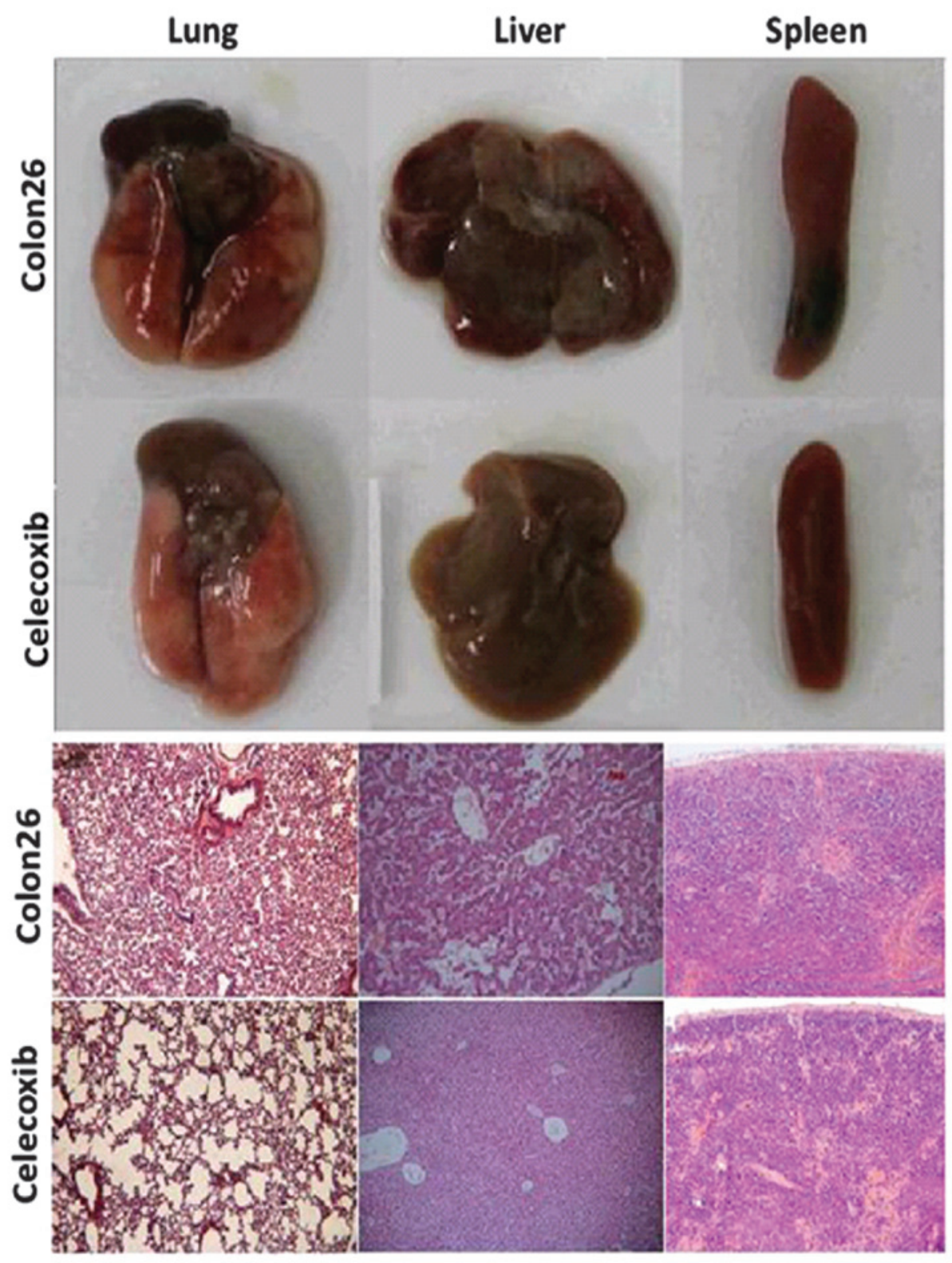

Figure 3. Celecoxib treatment exhibited effects on the inflammatory response in multiple organs. Images were captured and hematoxylin and eosin staining and evaluation conducted in lung, liver and spleen tissues. Magnification, x200.

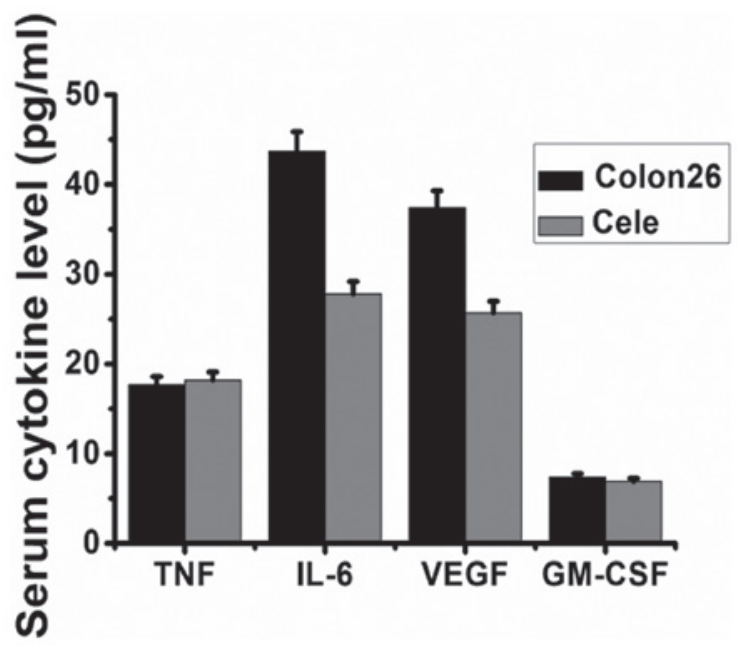

Figure 4. ELISA to determine the levels of inflammatory cytokines in the serum. Cele, celecoxib; TNF, tumor necrosis factor; IL, interleukin, VEGF, vascular endothelial growth factor; GM-CSF, granulocyte macrophage colony-stimulating factor. 
A

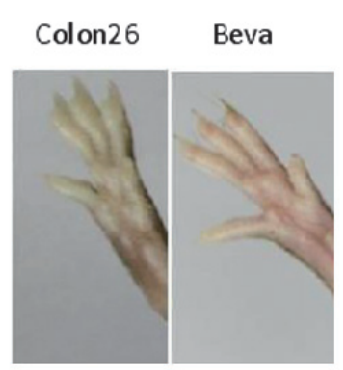

D

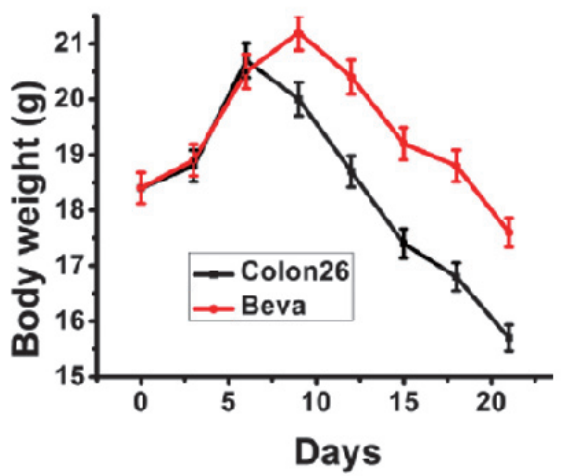

B

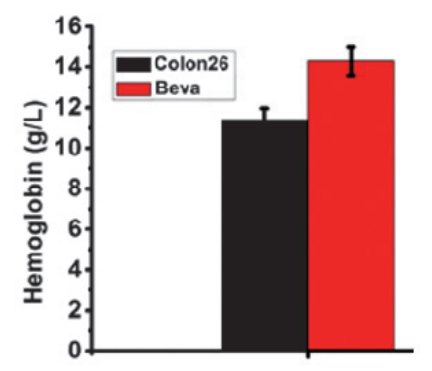

C

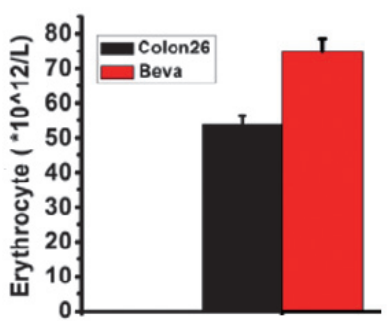

$\mathbf{E}$

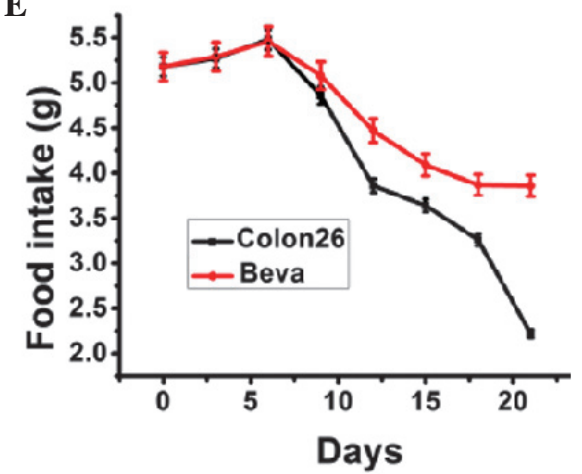

Figure 5. Anti-VEGF anitbody treatment (bevacizumab) for 10 days was able to reverse cachexia events. (A) Images of the paws from a representative mouse of each group were captured. Levels of (B) hemoglobin and (C) erythrocytes were determined. (D) Body weight of mice was measured every 3 days. (E) Food intake of mice was measured. Beva, bevacizumab.

\section{Discussion}

Cancer cachexia occurs in a large percentage of cases of advanced cancer, and reduces the strength of the patient, the effectiveness of cancer treatments and the quality of life $(1,2)$. In the current study, treatment with a selective COX-2 inhibitor, celecoxib, was demonstrated to attenuate symptoms of cachexia induced by colon 26 tumor injection, including severe anemia, loss of body weight and a reduction in food intake. Increasing evidence denotes that eicosanoids, such as prostaglandins are involved in the development of cachexia $(3,15,16,18)$, and other studies have suggested that the selective COX-2 inhibitor celecoxib is able to rapidly reverse this action (19-21). The observations of the present study, that celecoxib ameliorates cancer cachexia in colon 26 tumor model mice, is consistent with the results of the previous studies.

Although the precise mechanism underlying cancer cachexia remains to be fully elucidated, a current emerging hypothesis is that cachexia is caused predominantly by cytokines, either produced by cancer cells or released by the immune system in response to the presence of the cancer $(22,23)$. High serum levels of TNF- $\alpha$, IL-1 and IL- 6 have been observed in cancer patients, and the levels of these cytokines appear to correlate with the incidence of cancer cachexia $(24,25)$. Of these cytokines, IL- 6 has been demonstrated to enhance the reduction in fat and muscle tissue of cachectic animals (26). In the current study, administration of a COX-2 inhibitor significantly reduced the serum concentration of IL- 6 in tumor-bearing mice. Therefore, the COX-2 inhibitor exerts an anticachectic effect, potentially through the inhibition of IL-6 production in colon 26 tumor-bearing mice.

In the present study, administration of celecoxib also significantly reduced the serum concentration of VEGF in tumor-bearing mice. In order to further investigate the mechanisms underlying the anticachectic effect of the COX-2 inhibitor, bevacizumab, a VEGF antibody was used to treat the cachexia in mice, and the results indicated that anti-VEGF treatment was able to improve anemia, and increase food intake and body weight. VEGF is one of the most potent angiogenic factors (27) and it has been implicated in pathological angiogenesis associated with tumors and intraocular neovascular disorders (28). Another study suggested that tumor-derived VEGF is able to induce symptoms that resemble cancer cachexia and paraneoplastic syndromes (29). Kemik et al (30) demonstrated that tumor-derived VEGF induces cancer-associated systemic syndrome (CASS), a cancer-associated systemic syndrome with features similar to cachexia, by damaging the structure and function of multiple tissues and organs, and an anti-VEGF antibody reversed VEGF-induced CASS in tumor-bearing mice. COX-2 expression in cancer cells stimulates the production of VEGF, and the expression of VEGF by cancer cells can be inhibited using NSAIDs $(31,32)$. In the present study, it was demonstrated that COX-2 inhibitor treatment reduces the level of VEGF in serum, and anti-VEGF treatment attenuates cachectic events in tumor-bearing mice. These results provide a novel mechanistic insight into the role of the COX-2 inhibitor in the treatment of cachexia.

In conclusion, the present study demonstrated that the COX-2 inhibitor celecoxib exerts an anticachectic effect in 
colon 26 tumor-bearing mice through downregulation of VEGF. The present findings may provide an alternative mechanism by which COX-2 inhibitors can enhance the quality of life for patients suffering from cancer cachexia.

\section{Acknowledgements}

The current work was supported by the National Natural Science Foundation of China (grant no. 81272351) and the Natural Science Foundation of Shandong Province (grant nos. 2012G0021826 and 2R2012HM020).

\section{References}

1. Fearon K, Strasser F, Anker SD, et al: Definition and classification of cancer cachexia: an international consensus. Lancet Oncol 12: 489-495, 2011.

2. Kumar NB, Kazi A, Smith T, et al: Cancer cachexia: traditional therapies and novel molecular mechanism-based approaches to treatment. Curr Treat Options Oncol 11: 107-117, 2010.

3. Donohoe CL, Ryan AM and Reynolds JV: Cancer cachexia: mechanisms and clinical implications. Gastroenterol Res Pract 2011: 601434, 2011

4. Argilés JM, Busquets S, Toledo M and López-Soriano FJ: The role of cytokines in cancer cachexia. Curr Opin Support Palliat Care 3: 263-268, 2009.

5. Gupta SC, Kim JH, Kannappan R, et al: Role of nuclear factor $\kappa \mathrm{B}$-mediated inflammatory pathways in cancer-related symptoms and their regulation by nutritional agents. Exp Biol Med (Maywood) 236: 658-671, 2011.

6. Clària J: Publication of a special issue: resolution of acute inflammation and the role of lipid mediators. Scientific World Journal 10: 1553-1555, 2010

7. Tan BH, Ross JA, Kaasa S, et al; European Palliative Care Research Collaborative: Identification of possible genetic polymorphisms involved in cancer cachexia: a systematic review. J Genet 90: 165-177, 2011.

8. Argilés JM, Busquets S and López-Soriano FJ: Anti-inflammatory therapies in cancer cachexia. Eur J Pharmacol 668 (Suppl 1): S81-S86, 2011.

9. Batista ML Jr, Peres SB, McDonald ME, et al: Adipose tissue inflammation and cancer cachexia: possible role of nuclear transcription factors. Cytokine 57: 9-16, 2012.

10. Thiel A, Mrena J and Ristimäki A: Cyclooxygenase-2 and gastric cancer. Cancer Metastasis Rev 30: 387-395, 2011.

11. Turk HM, Camci C, Sevinc A, et al: Cyclooxygenase-2 expression is not a marker of poor survival in lung cancer. Asian Pac J Cancer Prev 13: 315-318, 2012.

12. Williams CS, Mann M and DuBois RN: The role of cyclooxygenases in inflammation, cancer and development. Oncogene 18: 7908-7916, 1999.

13. Morita Y, Hata K, Nakanishi M, et al: Cyclooxygenase-2 promotes tumor lymphangiogenesis and lymph node metastasis in oral squamous cell carcinoma. Int J Oncol, 41: 885-892, 2012.
14. Xin X, Majumder M, Girish GV, et al: Targeting COX-2 and EP4 to control tumor growth, angiogenesis, lymphangiogenesis and metastasis to the lungs and lymph nodes in a breast cancer model. Lab Invest 92: 1115-1128, 2012.

15. Tisdale MJ: Cachexia in cancer patients. Nat Rev Cancer 2: $862-871,2002$

16. Davis TW, Zweifel BS, O'Neal JM, et al: Inhibition of cyclooxygenase-2 by celecoxib reverses tumor-induced wasting. J Pharmacol Exp Ther 308: 929-934, 2004.

17. Yang SY, Yu H, Krygier JE, Wooley PH and Mott MP: High VEGF with rapid growth and early metastasis in mouse osteosarcoma model. Sarcoma 2007: 95628, 2007.

18. Evans WJ, Morley JE, Argilés J, et al: Cachexia: a new definition. Clin Nutr 27: 793-799, 2008.

19. Serhan CN, Krishnamoorthy S, Recchiuti A and Chiang N: Novel anti-inflammatory - pro-resolving mediators and their receptors. Curr Top Med Chem 11: 629-647, 2011.

20. Zhao QT, Yue SQ, Cui Z, et al: Potential involvement of the cyclooxygenase-2 pathway in hepatocellular carcinoma-associated angiogenesis. Life Sci 80: 484-492, 2007.

21. Seelaender M, Batista M Jr, Lira F, Silverio R and Rossi-Fanelli F: Inflammation in cancer cachexia: to resolve or not to resolve (is that the question?). Clin Nutr 31: 562-566, 2012.

22. Argilés JM, López-Soriano FJ and Busquets S: Mechanisms to explain wasting of muscle and fat in cancer cachexia. Curr Opin Support Palliat Care 1: 293-298, 2007.

23. Lawrence $\mathrm{T}$ and Fong $\mathrm{C}$ : The resolution of inflammation: anti-inflammatory roles for NF-kappaB. Int J Biochem Cell Biol 42: 519-523, 2010.

24. Patra SK and Arora S: Integrative role of neuropeptides and cytokines in cancer anorexia-cachexia syndrome. Clin Chim Acta 413: 1025-1034, 2012.

25. Laviano A, Molfino A, Seelaender M, et al: Carnitine administration reduces cytokine levels, improves food intake, and ameliorates body composition in tumor-bearing rats. Cancer Invest 29: 696-700, 2011.

26. Weidle UH, Klostermann S, Eggle D and Krüger A: Interleukin 6/interleukin 6 receptor interaction and its role as a therapeutic target for treatment of cachexia and cancer. Cancer Genomics Proteomics 7: 287-302, 2010.

27. Ferrara N, Gerber HP and LeCouter J: The biology of VEGF and its receptors. Nat Med 9: 669-676, 2003

28. Shojaei $\mathrm{F}$ and Ferrara N: Antiangiogenesis to treat cancer and intraocular neovascular disorders. Lab Invest 87: 227-230, 2007.

29. Krzystek-Korpacka M, Matusiewicz M, Diakowska D, et al: Acute-phase response proteins are related to cachexia and accelerated angiogenesis in gastroesophageal cancers. Clin Chem Lab Med 46: 359-364, 2008.

30. Kemik O, Sumer A, Kemik AS, et al: The relationship among acute-phase response proteins, cytokines and hormones in cachectic patients with colon cancer. World J Surg Oncol 8: 85, 2010.

31. Valcárcel M, Mendoza L, Hernández JJ, et al: Vascular endothelial growth factor regulates melanoma cell adhesion and growth in the bone marrow microenvironment via tumor cyclooxygenase-2. J Transl Med 9: 142, 2011.

32. Kim YY, Lee EJ, Kim YK, et al: Anti-cancer effects of celecoxib in head and neck carcinoma. Mol Cells 29: 185-194, 2010 . 